\title{
Development of \\ an Efficient Inverse Analysis Technique for Monitoring of Electroplating Current Density on Target Region in LSI Fabrication*
}

\author{
Yoshinao KISHIMOTO**, Yukiyoshi KOBAYASHI** and Toshihisa OHTSUKA** \\ ** Department of Mechanical Engineering, Tokyo City University \\ 1-28-1 Tamazutsumi, Setagaya-ku, Tokyo 158-8557, Japan \\ E-mail: ykishimo@tcu.ac.jp
}

\begin{abstract}
Our research focuses on the techniques for monitoring the current density distribution of the real-time electroplating. In the previous research, a novel technique to estimate electroplating current densities on plated surfaces from the data of the electric potentials in the plating cells has been developed for LSI fabrication technology. The technique described in this paper has been applied for a 2 dimensional (2D) analysis in our previous research work; however, the 2D approach limits the application of the proposed method. In this research, we applied the monitoring technique with 3 dimensional (3D) analysis. Although the inverse analysis using the 3D analysis tends to be large scale, it is important for the practical usage to monitor current density on target region accurately and efficiently. Therefore we have developed an efficient inverse analysis technique for monitoring of electroplating current density on the target region and verified the effectiveness of the proposed technique by using numerical simulations.
\end{abstract}

Key words : Inverse Problem, Large-Scale Integration, Electroplating, Real-Time Monitoring, Boundary Element Method, Model Selection

\section{Introduction}

Copper electroplating is widely used to fabricate Large-Scale Integration (LSI) whose components are from nanoscale wires to microscale bumps because of its excellent via/trench filling ability, good adhesion and lower process temperature and low $\operatorname{cost}^{(1)-(6)}$. During electroplating it is necessary for fine plating to monitor the plating current density to ensure uniformity and quality. The conventional method typically predicts the optimum conditions for electroplating using numerical simulation methods before the electroplating operation ${ }^{(7)-(10)}$. These numerical simulation methods require the preliminarily measured samples of the polarization curves which describe the relationships between the electric potentials and the current densities on the plated surfaces. Therefore the current density on the plated surface in the real-time situation is unable to be measured or estimated by the existing methods.

Our research focuses on the techniques for monitoring the current density distribution of the real-time plating and we have developed a novel technique to estimate electroplating current densities on plated surfaces from the data of the electric potentials in the plating cells for LSI fabrication technology ${ }^{(11)-(15)}$. An electric potential at a point can be measured by using a capillary inserted in the cell. On the other hand, evaluation of a physical quantity on a boundary from known values in the domain is an inverse problem. This proposed technique applies Tikhonov regularization ${ }^{(16)-(18)}$ on the mathematical model of the electric field derived by boundary element method (BEM) to solve this inverse problem. The regularization parameter is determined by using Kullback-Leibler divergence ${ }^{(19),(20)}$ which is the difference

${ }^{*}$ Received 21 June, 2011 (No. 11-0336) [DOI: 10.1299/jmmp.5.656]

Copyright (c) 2011 by JSME 
between the measurement error and the modeling error. Further we have conducted experimental verification and demonstrated the effectiveness of the presented method by actual measurements ${ }^{(12),(14)}$.

However we have applied with only 2 dimensional (2D) BEM analysis in our previous researches since the 2D BEM analysis is more accurate and calculates in less time than 3 dimensional (3D) BEM analysis ${ }^{(11)-(15)}$. The 2D BEM analysis can be applied on only axially uniform domains and axially symmetric domains. Hence it limits the application range of the proposed method.

In this research, we applied the monitoring technique of electroplating current densities by the inverse analysis of the electric potentials in the plating cells with the 3D BEM analysis. The inverse analysis using the 3D BEM analysis tends to be large scale. In contrast, it is important for the practical usage to monitor current density on target region accurately and efficiently. Therefore we have developed an efficient inverse analysis technique for monitoring of electroplating current density on the target region. This proposed technique consists of 2 steps: discard of low sensitive components in the observation equation for the inverse analysis and reutilization of the discarded components.

In this paper, first we describe the outlines of electroplating in LSI fabrication and the measurement method of electric potentials in a plating cell. Secondly, we explain the mathematical model of the electric field and the way to derive the observation equation for the inverse analysis. And then the efficient inverse analysis technique developed in this research is elaborated. Furthermore we have verified the proposed technique by using numerical simulations. Comparing the proposed method with simultaneous estimation of the entire current densities which is the method developed in our previous research ${ }^{(11)-(15)}$, those results show that the proposed method can estimate the current density on the target region more efficiently than the previous method.

\section{Outline of Electroplating in LSI Fabrication}

Figure 1 illustrates the model of an ordinary copper electroplating device to fabricate LSI. The DC power supply outside the plating cell provides direct current to the anode. The anode is a thick copper plate and the plating cell is filled with electrolyte whose major compoment is copper sulfate.

The LSI wafer is electroplated with copper after the TaN protection layer and the copper seed layer are formed by sputtering on the $\mathrm{SiO}_{2}$ insulating surface of the silicon wafer in an actual fabrication process (Fig. 2). The wafer is surrounded by the power supply terminals and the seed layer and the plating film formed on the seed layer become the cathodes.

Polarization curves which describe macroscopic relationships between current densities and electric potentials are often leveraged in electrical field computation of electroplating without considering complicate behaviors confined to the immediate vicinity of the anode surface and cathode surfaces and small trenches on the metal surfaces ${ }^{(7)}-(10)$.

\section{The Measurement Method of Electric Potentials in a Plating Cell}

Figure 3 illustrates the layout of the device for measuring electric potentials in a plating cell. The anode is copper source as raw material of wires or bumps and the cathode is LSI wafer. The cell is filled with electrolyte. The capillary is attached to the puddler and picks up the electrolyte at several locations. The reference electrode and the potentiometer measure the electric potential of the electrolyte at the capillary tip.

\section{Mathematical Model of the Electric Field}

\subsection{Mathematical Model}

The mathematical model for the inverse analysis is explained in this section. The boundary $\Gamma\left(\equiv \Gamma_{a}+\Gamma_{c}+\Gamma_{n}\right)$ surrounds the domain $\Omega$ by as shown in Figs. 1, 2 and 3, where $\Gamma_{a}$ and $\Gamma_{c}$ are the surface boundaries of the anode and the cathode, $\Gamma_{n}$ is the sidewall boundary of the 


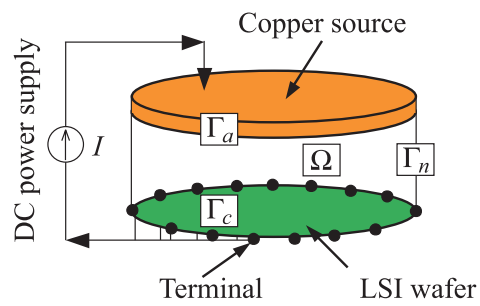

Fig. 1 The model of the LSI electroplating device

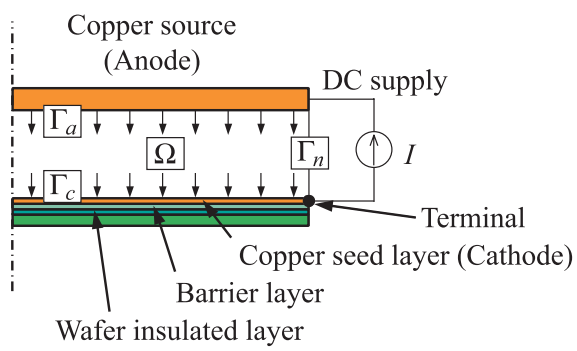

Fig. 2 The cross-sectional view of the device

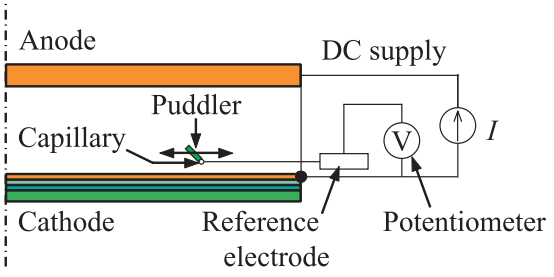

Fig. 3 The measurement method of electric potentials in a plating cell

plating cell where the current density is known and $\Omega$ is the domain filled with the electrolyte in the plating cell.

The electric potential in the domain is denoted by $u$ and the values of $u$ on the boundaries $\Gamma_{a}, \Gamma_{c}$ and $\Gamma_{n}$ are denoted by $u_{a}, u_{c}$ and $u_{n}$. The value of $u$ in the domain $\Omega$ excluding the boundary $\Gamma$ is denoted by $u_{i}$. Further the normal direction components of the current densities on the boundaries $\Gamma_{a}, \Gamma_{c}$ and $\Gamma_{n}$ are denoted by $j_{a}, j_{c}$ and $j_{n}(=0)$. The values of $j_{a}$ and $j_{c}$ are defined as positive values when those current densities are flowing into the domain $\Omega$ through the boundary $\Gamma$. Here $j_{a}$ dominates the decrease speed of the anode by electroplating and $j_{c}$ dominates the forming rate of the plating film. The electric potential $u$ obeys the following Laplace equation in the plating cell.

$$
\nabla^{2} u=0 \quad \text { in } \Omega
$$

The boundary condition of Eq. (1) is given as follows.

$$
\kappa \frac{\partial u}{\partial n}= \begin{cases}j_{a} & \text { on } \Gamma_{a} \\ j_{c} & \text { on } \Gamma_{c} \\ j_{n}=0 & \text { on } \Gamma_{n}\end{cases}
$$

where $\kappa$ is the electric conductivity of the electrolyte and $\partial / \partial n$ describes the normal differential.

Equation (1) is transformed into the following integral equation for the boundary $\Gamma$ by applying Green theorem after the both sides of Eq. (1) are multiplied by the fundamental solution of Laplace equation $v$ (ref. Eq. (4)) and integrated in the domain $\Omega$.

$$
u_{i}(\boldsymbol{p})=\int_{\Gamma}\left(v(\boldsymbol{p}, \boldsymbol{q}) \frac{\partial u(\boldsymbol{q})}{\partial n}-\frac{\partial v(\boldsymbol{p}, \boldsymbol{q})}{\partial n} u(\boldsymbol{q})\right) d \Gamma(\boldsymbol{q})
$$




$$
v(\boldsymbol{p}, \boldsymbol{q})=\frac{1}{4 \pi|\boldsymbol{p}-\boldsymbol{q}|}
$$

where $\boldsymbol{p}$ is the position vector of the measurement location and $\boldsymbol{q}$ is the position vector of the calculation location. Furthermore the following boundary integral equation is derived from Eq. (3) if the vector $\boldsymbol{p}$ points any location on the boundary $\Gamma$.

$$
c(\boldsymbol{p}) u(\boldsymbol{p})=\int_{\Gamma}\left(v(\boldsymbol{p}, \boldsymbol{q}) \frac{\partial u(\boldsymbol{q})}{\partial n}-\frac{\partial v(\boldsymbol{p}, \boldsymbol{q})}{\partial n} u(\boldsymbol{q})\right) d \Gamma(\boldsymbol{q})
$$

where $c(\boldsymbol{p})$ is the constant value determined by the shape of the boundary $\Gamma$ and the boundary integral in the right side of Eq. (5) is calculated as Cauchy's principal value integration.

\subsection{Observation Equation by Boundary Element Method}

The observation equation which associates the known value $u_{i}$ with the unknown values $u_{a}, u_{c}$ is derived from the mathematical model in this section. The following algebraic equation is derived by discretizing Eq. (5).

$$
H_{a} \boldsymbol{u}_{a}+H_{c} \boldsymbol{u}_{c}+H_{n} \boldsymbol{u}_{n}=G_{a} \boldsymbol{j}_{a}+G_{c} \boldsymbol{j}_{c}+G_{n} \boldsymbol{j}_{n}
$$

Similarly, the following algebraic equation is derived by discretizing Eq. (3).

$$
\boldsymbol{u}_{i}=G_{i a} \boldsymbol{j}_{a}+G_{i c} \boldsymbol{j}_{c}+G_{i n} \boldsymbol{j}_{n}-H_{i a} \boldsymbol{u}_{a}-H_{i c} \boldsymbol{u}_{c}-H_{i n} \boldsymbol{u}_{n}
$$

where $G_{a}, G_{c}, G_{n}, H_{a}, H_{c}, H_{n}$ are the known matrices determined by the discretized shape of the boundary $\Gamma$, and $G_{i a}, G_{i c}, G_{i n}, H_{i a}, H_{i c}, H_{i n}$ are the known matrices determined by the discretized shape of the boundary $\Gamma$ and the measurement locations of the electric potentials. $\boldsymbol{u}_{a}, \boldsymbol{u}_{c}, \boldsymbol{u}_{n}, \boldsymbol{j}_{a}, \boldsymbol{j}_{c}, \boldsymbol{j}_{n}$ are the vectors whose components are the values of $u_{a}, u_{c}, u_{n}, j_{a}, j_{c}, j_{n}$ at the nodes of the boundary elements and $\boldsymbol{u}_{i}$ is the vector whose components are the measured values of the electric potentials.

Since Eq. (6) is indeterminate for $\boldsymbol{u}_{a}, \boldsymbol{u}_{c}$ and $\boldsymbol{u}_{n}$, the reference potential $u_{d}(=0)$ is defined at the reference point on the boundary $\Gamma_{n}$ to solve the equation. Instead the value of the current density at the reference point (denoted by $j_{d}$ ) is given as a variable. Hence some of the terms in Eqs. (6) and (7) are decomposed as follows.

$$
\begin{gathered}
H_{n} \boldsymbol{u}_{n}=H_{n}^{\prime} \boldsymbol{u}_{n}^{\prime}+u_{d} \boldsymbol{h}_{d} \\
G_{n} \boldsymbol{j}_{n}=G_{n}^{\prime} \boldsymbol{j}_{n}^{\prime}+j_{d} \boldsymbol{g}_{d} \\
H_{i n} \boldsymbol{u}_{n}=H_{i n}^{\prime} \boldsymbol{u}_{n}^{\prime}+u_{d} \boldsymbol{h}_{i d} \\
G_{i n} \boldsymbol{j}_{n}=G_{i n}^{\prime} \boldsymbol{j}_{n}^{\prime}+j_{d} \boldsymbol{g}_{i d}
\end{gathered}
$$

Then Eq. (6) is solved for $\boldsymbol{u}_{a}, \boldsymbol{u}_{c}, \boldsymbol{u}_{n}^{\prime}$ and $j_{d}$ as follows.

$$
\left\{\begin{array}{c}
\boldsymbol{u}_{a} \\
\boldsymbol{u}_{c} \\
\boldsymbol{u}_{n}^{\prime} \\
j_{d}
\end{array}\right\}=\left[\begin{array}{llll}
H_{i a} & H_{i c} & H_{i c}^{\prime} & -\boldsymbol{g}_{i d}
\end{array}\right]\left[\begin{array}{ll}
G_{a} & G_{c}
\end{array}\right]\left\{\begin{array}{c}
\boldsymbol{j}_{a} \\
\boldsymbol{j}_{c}
\end{array}\right\}
$$

where $j_{n}=0$ and $u_{d}=0$ are considered.

Therefore the following equation is derived as the observation equation which associates the known values with the unknown values by using Eqs. (6), (7) and (12).

$$
\boldsymbol{b}=A \boldsymbol{x}
$$

where

$$
\boldsymbol{b}=\boldsymbol{u}_{i}
$$




$$
\begin{aligned}
A & =\left[\begin{array}{rr}
G_{i a} & G_{i c}
\end{array}\right]-\left[\begin{array}{cccc}
H_{i a} & H_{i c} & H_{i c}^{\prime} & -\boldsymbol{g}_{i d}
\end{array}\right] \\
& \cdot\left[\begin{array}{cccc}
H_{a} & H_{c} & H_{n}^{\prime} & -\boldsymbol{g}_{d}
\end{array}\right]^{-1}\left[\begin{array}{cc}
G_{a} & G_{c}
\end{array}\right] \\
\boldsymbol{x} & =\left\{\begin{array}{c}
\boldsymbol{j}_{a} \\
\boldsymbol{j}_{c}
\end{array}\right\}
\end{aligned}
$$

\section{Inverse Analysis Technique}

\subsection{Tikhonov Regularization}

The unknown values $j_{a}, j_{c}$ are estimated from the electric potential $u_{i}$ measured in actual experiments by using the observation equation (Eq. (13)). The observation equation is solved by applying Tikhonov regularization expressed in the following evaluation function $\Pi(x)$ on Eq. (13) to decrease the sensitivity of the measurement error.

$$
\Pi(\boldsymbol{x})=\|\boldsymbol{b}-A \boldsymbol{x}\|^{2}+\alpha\left\|\boldsymbol{x}-\boldsymbol{x}_{o}\right\|^{2}
$$

where $\alpha$ is Tikhonov regularization parameter and $\boldsymbol{x}_{o}$ is the vector of reference values. The averages of the current densities which are the quotients of the total current and the plated surface areas are utilized as the reference values for the vector $\boldsymbol{x}_{o}$.

The estimated value of $\boldsymbol{x}$ by Tikhonov regularization minimizes the evaluation function $\Pi(\boldsymbol{x})$. The solution $\hat{\boldsymbol{x}}$ is obtained by using the singular value decomposition of the matrix $A$ as follows.

$$
\hat{\boldsymbol{x}}=L \boldsymbol{b}+K \boldsymbol{x}_{o}
$$

where

$$
\begin{aligned}
& A=U S V^{T} \quad \text { (Singular Value Decomposition) } \\
& L=V\left(S^{T} S+\alpha E\right)^{-1} S^{T} U^{T} \quad(E \text { : Identity matrix) } \\
& K=\alpha V\left(S^{T} S+\alpha E\right)^{-1} V^{T}
\end{aligned}
$$

and the superscript $T$ denotes matrix transposition.

There are various criteria to determine the value of the regularization parameter $\alpha^{(16)-(18)}$. The value of $\alpha$ is determined as follows in this research. The value of $\hat{\boldsymbol{x}}(\alpha)$ is obtained by Eq. (18) when a certain value is assigned to $\alpha$. Furthermore the variance of the modeling error $\hat{\sigma}^{2}(\alpha)$ is obtained from the estimated solution $\hat{\boldsymbol{x}}(\alpha)$ by Eq. (13) as follows.

$$
\hat{\sigma}^{2}(\alpha)=\frac{\|\boldsymbol{b}-A \hat{\boldsymbol{x}}(\alpha)\|^{2}}{m}
$$

where $m$ is the dimension of the vector $\boldsymbol{b}$. Then the value of $\alpha$ which minimizes the following Kullback-Leiblar divergence $\operatorname{KLD}(\alpha)$ is employed.

$$
\mathrm{KLD}(\alpha)=\log \hat{\sigma}^{2}(\alpha)+\frac{2 \sigma^{2}}{m \hat{\sigma}^{2}(\alpha)} \sum_{i=1}^{s} \frac{\lambda_{i}^{2}}{\lambda_{i}^{2}+\alpha}
$$

where $\sigma^{2}, s$ and $\lambda_{i}$ are the variance of the measurement error, the number of the singular values and $i$ th order of the singular value of $A$, respectively. Kullback-Leiblar divergence $\operatorname{KLD}(\alpha)$ means the difference between the variance of the measurement error $\sigma^{2}$ and the variance of the modeling error $\hat{\sigma}^{2(19),(20)}$. The value of $\alpha$ which minimizes Eq. (23) is calculated by using the golden section method. 


\subsection{An Efficient Calculation Method of the Inverse Analysis}

The dimension of the unknown value vector $\boldsymbol{x}$ in Eq. (13) becomes large when the estimated domain or its resolution is large in the case that 3D domain analysis is operated. If the dimension of the unknown value vector $\boldsymbol{x}$ is large, the problem becomes a large scale inverse problem and an enormous amount of calculation to solve the inverse problem is necessary. Moreover measurement data $\boldsymbol{b}$ in Eq. (13) whose quantity is more than or equal to the dimension of the unknown value vector $\boldsymbol{x}$ is also necessary to obtain more accurate solutions of the unknown values. This means the actual measurement requires time and effort.

To reduce the calculation amount of the inverse analysis and the necessary quantity of the actual measurement data, an efficient calculation method of the inverse analysis for the large scale inverse problem has been developed in this research. In the actual monitoring of the electroplating current density, it is not necessarily the case that the entire current density is necessary and the estimation of the current density on a target region such as the cathode edge where high current density flows into is enough. Therefore only the current density on the target region, i.e., the target component of $\boldsymbol{x}$ (denoted by $x_{l}$ ) is estimated efficiently in the proposed method. The proposed method discards the components of the unknown value vector $\boldsymbol{x}$ and the observed vector $\boldsymbol{b}$ whose sensitivities for estimation of the value of the target component $x_{l}$ are low by the following procedure.

5.2.1. Discard of Low Sensitive Components When the dimension of the unknown value vector $\boldsymbol{x}$ is $n$ and the $i, j$ th component of the matrix $A$ is expressed as $a_{i j}$, Eq. (13) is written for each component as follows.

$$
\left\{\begin{array}{c}
b_{1} \\
\vdots \\
b_{k} \\
\vdots \\
b_{m}
\end{array}\right\}=\left[\begin{array}{ccccc}
a_{11} & \ldots & a_{1 l} & \ldots & a_{1 n} \\
\vdots & \ddots & \vdots & & \vdots \\
a_{k 1} & \ldots & a_{k l} & \ldots & a_{k n} \\
\vdots & & \vdots & \ddots & \vdots \\
a_{m 1} & \ldots & a_{m l} & \ldots & a_{m n}
\end{array}\right]\left\{\begin{array}{c}
x_{1} \\
\vdots \\
x_{l} \\
\vdots \\
x_{n}
\end{array}\right\}
$$

where let the $k, l$ th component $a_{k l}$ be the maximum component in the $l$ th column of the matrix $A$. Then $b_{k}$ which denotes the $k$ th component of the vector $\boldsymbol{b}$ has the highest sensitivity for the target component $x_{l}$. On the other hand, the small components in the matrix $A$ have low sensitivities for the vector $\boldsymbol{x}$ so that the small components compared to $a_{k l}$ in $k$ th row are discarded in the column direction at first. In the row direction, the rows whose $l$ th column components $a_{i l}$ are small compared to $a_{k l}$ are discarded. Figure 4 illustrates the procedure to discard the low sensitive components in the matrix $A$.

The criteria to discard the small components are defined as follows in this research. In the column direction,

$$
\left|a_{k j}\right|\left\{\begin{array}{ccc}
\geq \eta\left|a_{k l}\right| & \rightarrow & \text { Utilize the } j \text { th column } \\
<\eta\left|a_{k l}\right| & \rightarrow & \text { Discard the } j \text { th column }
\end{array}\right.
$$

and in the row direction,

$$
\left|a_{i l}\right|\left\{\begin{array}{lll}
\geq \zeta\left|a_{k l}\right| & \rightarrow & \text { Utilize the } i \text { th row } \\
<\zeta\left|a_{k l}\right| & \rightarrow & \text { Discard the } i \text { th row }
\end{array}\right.
$$

where $\eta$ and $\zeta$ are the ratios whose are obtained by the number of the measurement points in the actual monitoring and the unknown components estimated simultaneously with the target component. Then $\eta$ and $\zeta$ satisfy the following inequalities.

$$
\frac{\min \left|a_{k j}\right|}{\left|a_{k l}\right|} \leq \eta \leq \frac{\max \left|a_{k j}\right|}{\left|a_{k l}\right|} \text { and } \frac{\min \left|a_{i l}\right|}{\left|a_{k l}\right|} \leq \zeta \leq \frac{\max \left|a_{i l}\right|}{\left|a_{k l}\right|}
$$


(1) Discarding the small components in the column direction

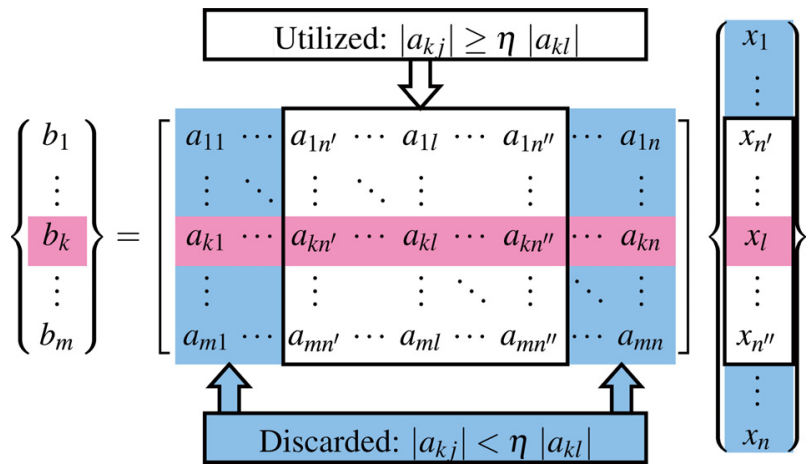

(2) Discarding the small components in the row direction

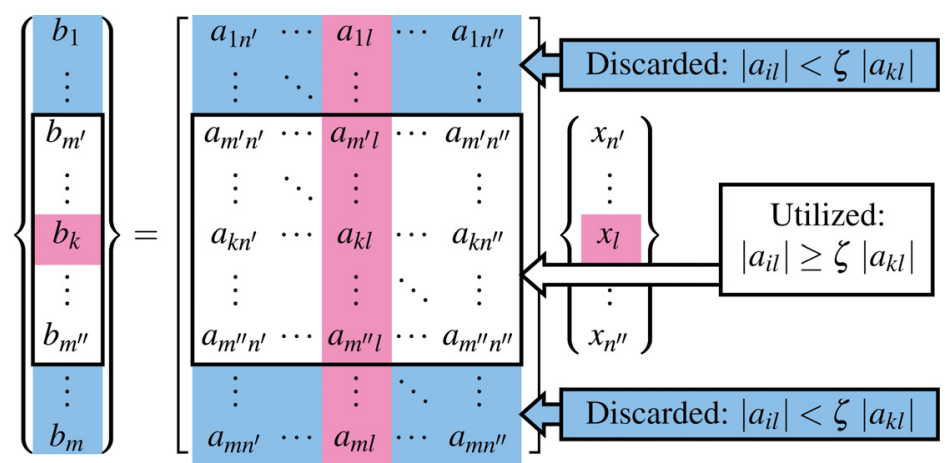

\section{(3) Completed}

$$
\left\{\begin{array}{c}
b_{m^{\prime}} \\
\vdots \\
b_{k} \\
\vdots \\
b_{m^{\prime \prime}}
\end{array}\right\}=\left[\begin{array}{ccccc}
a_{m^{\prime} n^{\prime}} & \cdots & a_{m^{\prime} l} & \cdots & a_{m^{\prime} n^{\prime \prime}} \\
\vdots & \ddots & \vdots & & \vdots \\
a_{k n^{\prime}} & \cdots & a_{k l} & \cdots & a_{k n^{\prime \prime}} \\
\vdots & & \vdots & \ddots & \vdots \\
a_{m^{\prime \prime} n^{\prime}} & \cdots & a_{m^{\prime \prime} l} & \cdots & a_{m^{\prime \prime} n^{\prime \prime}}
\end{array}\right]\left\{\begin{array}{c}
x_{n^{\prime}} \\
\vdots \\
x_{l} \\
\vdots \\
x_{n^{\prime \prime}}
\end{array}\right\}
$$

Fig. 4 The procedure to discard the low sensitive components in the matrix $A$

5.2.2. Reutilization of the Discarded Components Only discarding the columns is equivalent to assuming that $x_{j}=0\left(j \notin D^{c o l}: D^{c o l}\right.$ denotes the set of the columns of the matrix $A$ utilized by the above procedure). However the actual current density is not usually zero so that this assumption is a constraint condition. This research reutilizes the discarded components as follows to give tolerance for the inverse analysis.

$$
\left\{\begin{array}{c}
b_{m^{\prime}} \\
\vdots \\
b_{k} \\
\vdots \\
b_{m^{\prime \prime}}
\end{array}\right\}=\left[\begin{array}{ccccccc}
a_{m^{\prime} n^{\prime}} & \cdots & a_{m^{\prime} l} & \cdots & a_{m^{\prime} n^{\prime \prime}} & \sum_{j \in \bar{D}_{a}^{c o l}} a_{m^{\prime} j} & \sum_{j \in \bar{D}_{c}^{c o l}} a_{m^{\prime} j} \\
\vdots & \ddots & \vdots & & \vdots & \vdots & \vdots \\
a_{k n^{\prime}} & \cdots & a_{k l} & \cdots & a_{k n^{\prime \prime}} & \sum_{j \in \bar{D}_{a}^{c o l}} a_{k j} & \sum_{j \in \bar{D}_{c}^{c o l}} a_{k j} \\
\vdots & & \vdots & \ddots & \vdots & \vdots & \vdots \\
a_{m^{\prime \prime} n^{\prime}} & \cdots & a_{m^{\prime \prime} l} & \cdots & a_{m^{\prime \prime} n^{\prime \prime}} & \sum_{j \in \bar{D}_{a}^{c o l}} a_{m^{\prime \prime} j} & \sum_{j \in \bar{D}_{c}^{c o l}} a_{m^{\prime \prime} j}
\end{array}\right]\left\{\begin{array}{c}
x_{n^{\prime}} \\
\vdots \\
x_{l} \\
\vdots \\
x_{n^{\prime \prime}} \\
x_{a} \\
x_{c}
\end{array}\right\}
$$

where $m^{\prime}, \cdots, k, \cdots, m^{\prime \prime}$ and $n^{\prime}, \cdots, l, \cdots, n^{\prime \prime}$ are the rows and the columns utilized by the discarding procedure. $\bar{D}_{a}^{c o l}$ and $\bar{D}_{c}^{\text {col }}$ are the set of the discarded columns of the matrix $A$ 
corresponding to the anode elements and the cathode elements. $x_{a}$ and $x_{c}$ are the unknown current densities on the BEM elements corresponding to the discarded columns, and they are estimated by the inverse analysis. Using Eq. (29) instead of Eq. (24) for the inverse analysis is equivalent to assuming that the current densities on the anode area and the cathode area corresponding to the discarded columns are uniform.

\section{Verification by Numerical Experiment}

\subsection{Conditions of the Numerical Experiments}

Numerical experiments were performed to verify the effectivity of the proposed method. The 3D BEM model as shown in Fig. 5 is used in the experiments. The electroplating cell is a cylinder whose diameter and height are $300 \mathrm{~mm}$ and $10 \mathrm{~mm}$, and its top side is the anode surface and its bottom side is the cathode surface. The existence of the puddler and the capillary is ignored in this model. In Fig. 5, the elements are constant triangle elements and the total number of the elements is 4500 . The number of elements for the anode surface and the cathode surface are 1350 each, and the number of the sidewall boundary is 1800 . Therefore the number of unknown values is 2700 in the inverse analysis using the 3D BEM model as shown in Fig. 5, and this inverse problem becomes large scale.

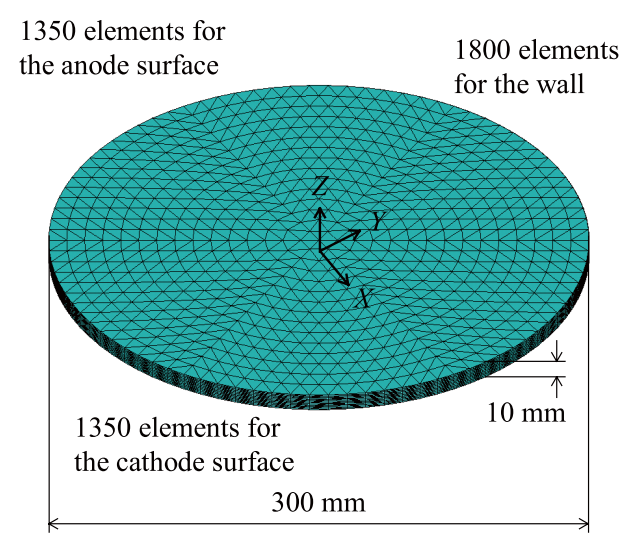

Fig. 5 The BEM model for 3D domain analysis

The average of the current density is $100 \mathrm{~A} / \mathrm{m}^{2}$ and the correct current densities on the anode surface and the cathode surface are calculated by the conventional numerical simulation method $^{(7)-(10)}$. Figure 6 shows the sample polarization curve of copper in copper sulfate used in this calculation. The electric potentials at the measurement points are also obtained by the conventional method. The 2D BEM model as shown in Fig. 7 is also used to obtain more accurate phantom data of the electric potentials. The reference points of the electric potentials are set at $R=\sqrt{X^{2}+Y^{2}}=150 \mathrm{~mm}, Z=0.5 \mathrm{~mm}$ on the sidewall elements to preserve the axial symmetry of the electric field. Then the current densities on the anode surface and the cathode

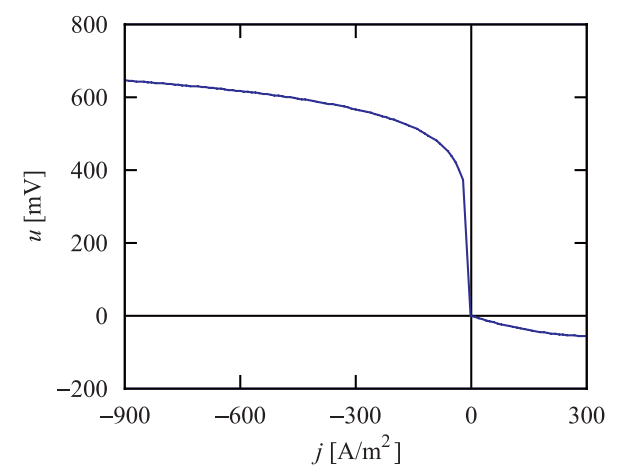

Fig. 6 The polarization curve of copper in copper sulfate 


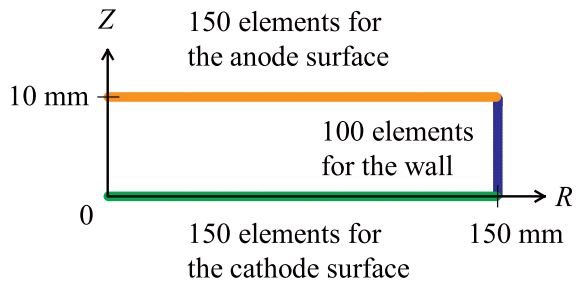

Fig. 7 The BEM model for 2D axially symmetric analysis

surface are estimated from the electric potentials by the proposed method. The measurement points of the electric potentials set in the numerical experiments are described below.

\subsection{Results of the 3D Domain Analysis}

6.2.1. Simultaneous Estimation of the Entire Unknown Values Figure 8 shows the locations of the measurement points for the simultaneous estimation of the entire unknown values. The measurement points are distributed as Fig. 8 at $Z=1 \mathrm{~mm}$ and $Z=9 \mathrm{~mm}$. The interval of the measurement points along a line such as the $X$-axis or the $Y$-axis is $2.5 \mathrm{~mm}$ and the total number of them is 2834 .

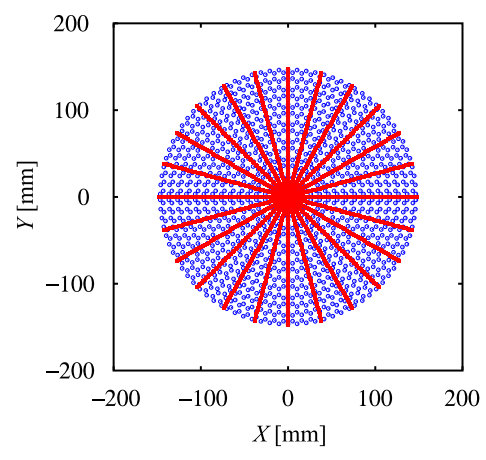

Center of BEM element $\bigcirc$

Measurement point

Fig. 8 The locations of the measurement points for the entire estimation

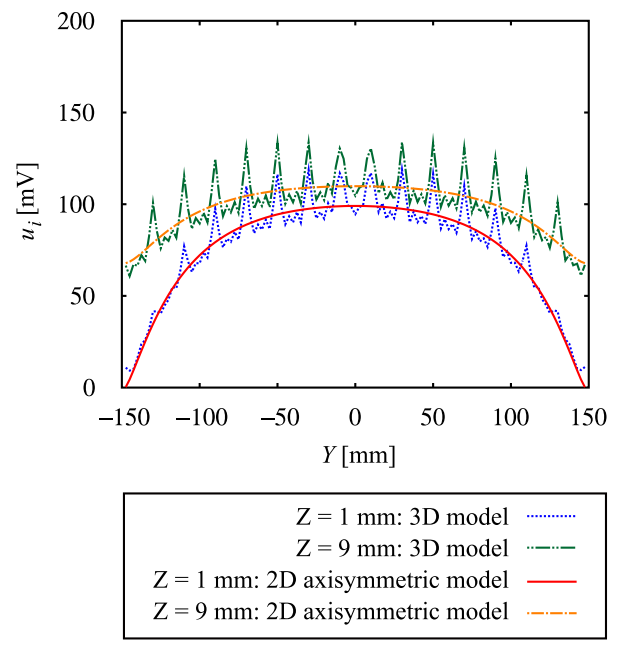

Fig. 9 The phantom data of the measured electric potentials 


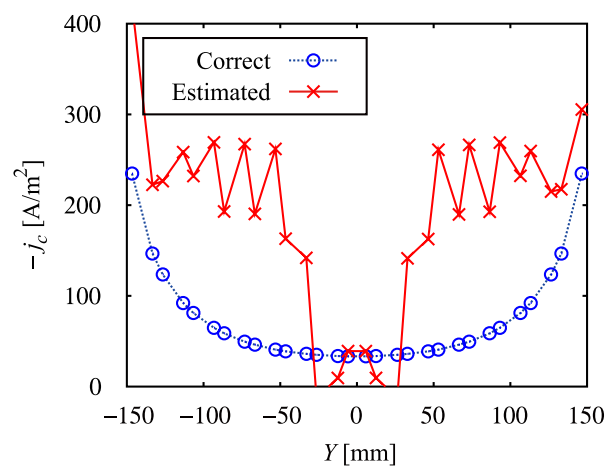

Fig. 10 The current densities on the cathode surface by the entire estimation

Figure 9 shows the electric potentials at the measurements points along the $Y$-axis calculated by the conventional numerical simulation. The data of the electric potentials at the other points are similar to the data in Fig. 9. The phantom data calculated by the 2D axially symmetric analysis are extended to the 3D domain in Fig. 9. From Fig. 9, the accuracy of the electric potentials calculated by the $3 \mathrm{D}$ domain analysis is low since the element discretization is still not enough, and this causes the estimation error.

Figure 10 shows the current densities on the cathode surface along the $Y$-axis estimated from the all data of the electric potentials calculated by the 2D axially symmetric analysis. The correct current densities by the conventional numerical simulation are also shown in Fig. 10. We set the variance of the measurement error $\sigma^{2}=25 \mathrm{mV}^{2}$ (thus $\sigma=5 \mathrm{mV}$ ) to apply the inverse analysis. From Fig. 10, the estimation of the current densities was failed in this case. The reason is that the variance of the measurement error $\sigma^{2}$ set for the inverse analysis was still smaller than the variance of the estimation error $\hat{\sigma}^{2}$ since the regularization of the estimated solution was not enough. In addition, the size of the matrix $A$ is $2834 \times 2700$ and this analysis takes computation time. Therefore seeking the sufficient value of $\sigma^{2}$ for the regularization also takes enormous time.

6.2.2. Efficient Estimation of the Target Unknown Value Figures 11 and 12 show the locations of the measurement points for the estimation and the current densities on the cathode surface along the $Y$-axis estimated by the proposed method when the coordinate of the target boundary element was set as $(0,147,0) \mathrm{mm}$. We set the size of the refined matrix $A$ as $50 \times 10$ and $\sigma^{2}=25 \mathrm{mV}^{2}$ (thus $\sigma=5 \mathrm{mV}$ ) to apply the proposed method in this experiment.

The 1572 candidate points were located with $2.5 \mathrm{~mm}$ interval near the target element at $Z=1 \mathrm{~mm}$ and $Z=9 \mathrm{~mm}$, and the 50 measurement points were selected by the proposed method as shown in Fig. 11. Also the 10 boundary elements were selected by the proposed
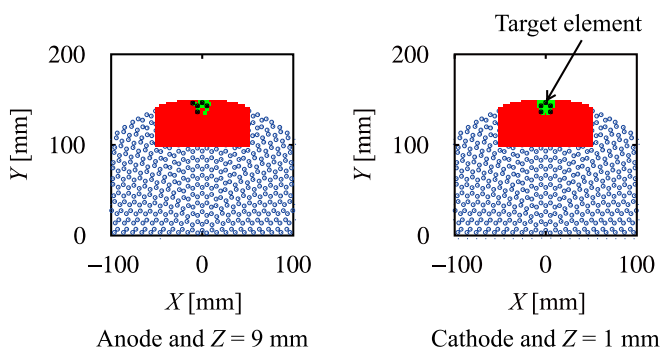

$$
\begin{array}{|c|c|}
\hline \multicolumn{3}{|c|}{\text { Center of BEM element }} \\
\text { Utilized } \otimes \text { Discarded } & \\
\text { Measurement point } & \\
\text { Utilized } \square \text { Discarded } & \\
\hline
\end{array}
$$

Fig. 11 The locations of the measurement points for the efficient estimation: the estimation target is $(0,147,0) \mathrm{mm}$ 


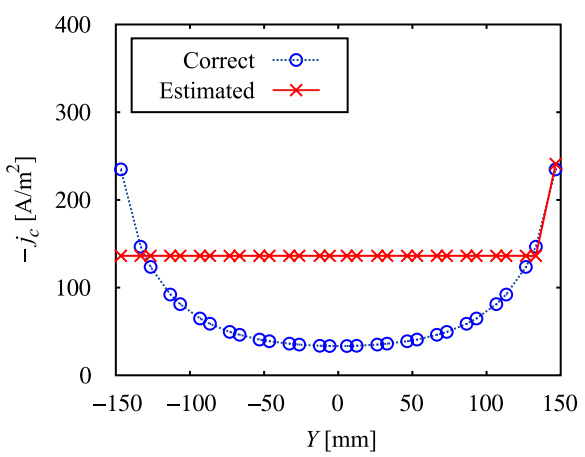

Fig. 12 The current densities on the cathode surface by the efficient estimation: the estimation target is $(0,147,0) \mathrm{mm}$
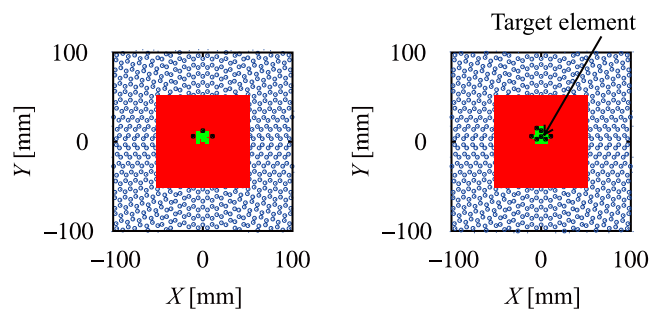

Anode and $Z=9 \mathrm{~mm}$

Cathode and $Z=1 \mathrm{~mm}$

\begin{tabular}{|c|}
\hline Center of BEM element \\
Utilized $\otimes$ Discarded \\
Measurement point \\
Utilized $\square$ Discarded \\
\hline
\end{tabular}

Fig. 13 The locations of the measurement points for the efficient estimation: the estimation target is $(0,5.77,0) \mathrm{mm}$

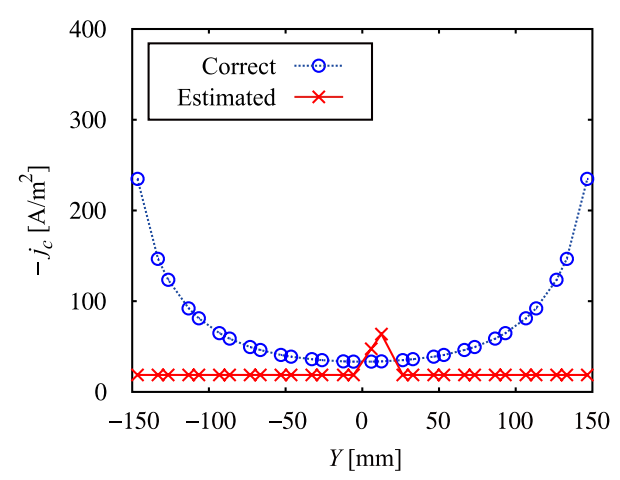

Fig. 14 The current densities on the cathode surface by the efficient estimation: the estimation target is $(0,5.77,0) \mathrm{mm}$

method as shown in Fig. 11. Then $\eta=0.328$ and $\zeta=0.304$. From Fig. 12, the current density at the target element was estimated successfully since the correct value $-j_{c}=235 \mathrm{~A} / \mathrm{m}^{2}$ and the estimated value $-\hat{j}_{c}=241 \mathrm{~A} / \mathrm{m}^{2}$. The size of the matrix $A$ is small $(50 \times 10)$ therefore the proposed method can estimate the target unknown value efficiently.

We conducted another experiment in the same condition to verify the proposed method. Figures 13 and 14 show the locations of the measurement points for the estimation and the current densities on the cathode surface along the $Y$-axis estimated by the proposed method when the coordinate of the target boundary element was set as $(0,5.77,0) \mathrm{mm}$. We set the size of the refined matrix $A$ as $50 \times 10$ and $\sigma^{2}=25 \mathrm{mV}^{2}$ (thus $\sigma=5 \mathrm{mV}$ ) to apply the proposed method in this experiment. 
The 3362 candidate points were located with $2.5 \mathrm{~mm}$ interval near the target element at $Z=1 \mathrm{~mm}$ and $Z=9 \mathrm{~mm}$, and the 50 measurement points were selected by the proposed method as shown in Fig. 13. Also the 10 boundary elements were selected by the proposed method as shown in Fig. 13. Then $\eta=0.558$ and $\zeta=0.455$. From Fig. 14, the current density at the target element was estimated successfully since the correct value $-j_{c}=33.4 \mathrm{~A} / \mathrm{m}^{2}$ and the estimated value $-\hat{j}_{c}=47.5 \mathrm{~A} / \mathrm{m}^{2}$. Therefore the proposed method is expected to be able to estimate any unknown value at any target point. Then the proposed method is expected to be able to estimate the entire unknown values more efficiently than the simultaneous estimation if the proposed method is applied on the each unknown value.

\section{Conclusion}

In this paper, we have developed an efficient inverse analysis technique for monitoring of electroplating current density on target region in LSI fabrication. The objective is to establish the estimation method of the current density on the target region in 3D domain efficiently due to the fact that the 3D analysis tends to be large scale. This proposed technique consists of 2 steps in addition to the inverse analysis developed in our previous research: discard of low sensitive components in the observation equation for the inverse analysis and reutilization of the discarded components.

We have also performed the numerical experiments to verify the effectiveness of the proposed method. The simultaneous estimation of the entire current densities which is the method developed in our previous research took computation time and the estimation was failed in this numerical experiment. On the other hand, the proposed method could estimate the current density on the target region more efficiently than the previous method. Those results show that the proposed method is expected to be able to estimate any unknown value at any target point. Then the proposed method is expected to be able to estimate the entire unknown values more efficiently than the simultaneous estimation if the proposed method is applied on the each unknown value.

Our future works are developments of criteria to determine the necessary size of the matrix in the observation equation and more effective reutilization of the discarded matrix components by the proposed technique. In particular, the former is expected to enable efficient measurements in the actual usage owing to that the criteria can determine the minimum number of the measurement points for the estimation.

\section{References}

( 1 ) Kodera, T., Okubo, T. and Kondo, K. "Uniformity Formation of Pannel Plating by using Convexity -Modification of Cathode Holder." Journal of Japan Institute of Electronics Packaging, Vol.7, No.2 (2004), pp.141-146.

( 2 ) Okubo, T., Kodera, T. and Kondo, K. "Uniformity of Patterned Copper Electrodeposit Thickness by using Auxiliary Electrode -Placement of Electrodes at the Edges of the Pattern." Journal of Japan Institute of Electronics Packaging, Vol.7, No.4 (2004), pp.322-327.

( 3 ) Satake, T. and Noji, N. "Development of an Electron Beam Inspection System (EBeye), 1st Report.” Ebara Engineering Review, No.207 (2005), pp.15-20.

( 4 ) Hodai, M., Nakada, T. and Kanda, H. "Cu Interconnect Plating System.” Ebara Engineering Review, No.207 (2005), pp.30-33.

( 5 ) Kuriyama, F., Minami, Y. and Kimura, M. "Bump Plating System.” Ebara Engineering Review, No.207 (2005), pp.34-38.

( 6 ) Terao, K., Karimata, T. and Sobukawa, H. "Development of an Electron Beam Inspection System (EBeye), 2nd Report.” Ebara Engineering Review, No.211 (2006), pp.2127.

( 7 ) Aoki, S., Amaya, K., Takazawa, H. and Miyasaka, M. "Boundary Element Simulation of 
Electroplating on Silicon Wafer." The Journal of the Surface Finishing Society of Japan, Vol.51, No.4 (2000), pp.425-430.

( 8 ) Aoki, S., Amaya, K., Abe, K. and Miyasaka, M. "Optimization of Electroplating on Silicon Wafer." Transactions of the Japan Society of Mechanical Engineers, Series A, Vol.68, No.666 (2002), pp.133-138.

( 9 ) Hayabusa, K., Takemura, T. and Amaya, K. "Development of a Plating Simulation Method for 3-Dimensional Models." Proceedings of the 2006 Annual Meeting of JSME/MMD, Vol.06, No.4 (2006-08), pp.409-410.

(10) Hayabusa, K., Takemura, T. and Amaya, K. "Optimization of the Shape of Plating Bath to Planarize Thickness of the Distribution in Semiconductor Plating." Proceedings of the 2006 JSME Millennium, Vol.06, No.6 (2006-09), pp.105-106.

(11) Kishimoto, Y. and Amaya, K. "Estimation Method of Copper Electroplating Current Density Distribution using Measurement Data of Electric Potentials in Plating Bath." Transactions of the Japan Society of Mechanical Engineers, Vol.74, No.745 (2008), pp.1204-1211.

(12) Kishimoto, Y., Amaya, K. and Hayabusa, K. "An Estimation Method of Copper Electroplating Current Densities by Inverse Analysis of Electric Potentials in Cells and Its Experimental Verification.” Transactions of the Japan Society of Mechanical Engineers, Vol.75, No.757 (2009), pp.1239-1246.

(13) Kishimoto, Y., Amaya, K., Hayabusa, K. and Onishi, Y. "Estimation of Electroplating Current Densities on LSI Wafer using Measurement Information of Electric Potentials in Cells." Proceedings of the 22nd Computational Mechanics Conference, No.09-22 (2009), No.802.

(14) Kishimoto, Y., Amaya, K. and Hayabusa, K. "An Estimation Method of Electroplating Current Densities in LSI Fabrication Technology by Inverse Analysis of Electric Potentials in Cells." Proceedings of the 8th IEEE Conference on Sensors, No.09-08 (2009), pp.53-56.

(15) Kishimoto, Y. and Amaya, K. "Development of a Real-Time Monitoring Method of Electroplating Current Densities in LSI Fabrication by Inverse Analysis of Electric Potentials." Proceedings of Asian Pacific Conference for Materials and Mechanics 2009, No.09-01 (2009), No.a236.

(16) Hansen, P.C. "Analysis of Discrete Ill-Posed Problems by Means of the L-Curve." Society for Industrial and Applied Mathematics Review, Vol.34, No.4 (1992), pp.561-580.

(17) Belge, M., Kilmer, M. and Miller, E.L. "Simultaneous Multiple Regularization Parameter Selection by Means of the L-Hypersurface with Applications to Linear Inverse Problems Posed in the Wavelet Transform Domain." Bayesian Inference for Inverse Problems, Vol.3459 (1998), pp.328-336.

(18) Oraintara, S., Karl, W.C., Castanon, D.A. and Nguyen, T.Q. "A Method for Choosing the Regularization Parameter in Generalized Tikhonov Regularized Linear Inverse Problems." Proceedings of the 2000 IEEE International Conference on Image Processing, Vol.1 (2000), pp.93-96.

(19) Kullback, S. and Leibler, R.A. "On Information and Sufficiency." The Annals of Mathematical Statistics, Vol.22, No.1 (1951), pp. 79-86.

(20) Burnham, K.P. and Anderson, D.R. Model Selection and Multimodel Inference: A Practical Information-Theoretic Approach, (2000), pp.49-97, Springer. 